http://jmscr.igmpublication.org/home/ ISSN (e)-2347-176x ISSN (p) 2455-0450

crossref DOI: https://dx.doi.org/10.18535/jmscr/v8i12.19

\title{
Study of Clinical Profile of Multiple Myeloma and Impact of Novel Therapies on Quality of Life
}

\author{
Authors \\ Shruthi Vitalarao Kulkarni ${ }^{1 *}$, Sunanda Amonkar ${ }^{2}$ \\ ${ }^{1}$ Senior Resident, Dept. of General Medicine \\ ${ }^{2}$ Associate Professor, Dept. of General Medicine \\ *Corresponding Author \\ Shruthi Vitalarao Kulkarni
}

\begin{abstract}
Multiple myeloma is one of the common plasma cell dyscrasia. it has heterogenous clinical presentation, and it can involve multiple organs. Its prevalence is more common in males than in females, and in black population. Etiology of multiple myeloma include radiation exposure, exposure to variety of carcinogens, chronic antigenic stimulation, autoimmunity.

There has been a significant change in treatment modality of multiple myeloma. The median survival of multiple myeloma patients was less than a year before the introduction of alkylating agents. Introduction of Melphalan, highdose chemotherapy and stem cell have demonstrated a survival advantage compared with patients undergoing conventional chemotherapy. The introduction of Thalidomide was a major milestone in the treatment of multiple myeloma, and its analog Lenalidomide and the proteasome inhibitor Bortezomib have expanded the therapeutic spectrum for multiple myeloma. With development of newer chemotherapy regimens, quality of life has improved, but at the same time these newer regimens are associated with specific side effects.

Keywords: Multiple myeloma, staging, electrophoresis, chemotherapy, relapse.
\end{abstract}

\section{Introduction}

Multiple myeloma is a neoplastic disorder of plasma cells characterized by a pentad of anemia, renal failure, M- protein in the serum or urine or both, osteolytic bone lesions and/ or bone pain and hypercalcemia ${ }^{1}$ Multiple myeloma affects 1 to 5 per 100000 people each year worldwide with a higher incidence in the west 1 . It accounts for $1 \%$ of all malignancies and $10 \%$ of all hematological malignancies ${ }^{1}$. After monoclonal gammopathy of undetermined significance, multiple myeloma is the most common plasma cell dyscrasia.

The median survival of multiple myeloma patients was less than a year before the introduction of alkylating agents; the introduction of Melphalan in the 1960s, resulted in significantly improved survival. In 1980s, high-dose chemotherapy and stem cell rescue were introduced, and randomized trials since then have demonstrated a survival advantage of transplant patients compared with patients undergoing conventional chemotherapy. The introduction of Thalidomide was a major milestone in the treatment of myeloma, and its analog Lenalidomide and the proteasome inhibitor Bortezomib have expanded the therapeutic spectrum for myeloma. Incorporation of these novel agents and supportive care has resulted in a significant shift in the treatment of myeloma, with their use earlier in the disease course. 


\section{Materials and Methods}

Our study was a prospective observational study. A total of 30 patients were enrolled in the study. Patients were selected from the in-patient departments of tertiary health care center. This study was conducted over two years starting from October 2016 to September 2018.We included patients of either gender with age between 18 to 80 years who satisfied the diagnostic criteria of multiple myeloma and willing to give the written informed consent for the study. We excluded patients with lytic lesions secondary to other known causes. Patients who satisfied the diagnostic criteria of multiple myeloma were selected from the in-patient department of tertiary care center

A detailed history was taken and a thorough clinical examination was done for all patients enrolled in the study. Necessary bio-chemical tests were performed and bone marrow findings were noted at presentation. Patients were followed up and at each visit, any fresh complaints were enquired for. Patients were assessed for treatment response and any treatment related complications were noted. Necessary tests were repeated as and when required. Data was analyzed using SPSS software version 17. To assess the effect of novel therapies, comparison was made with earliest studies. Student $t$ test was used.

\section{Observations and Results}

\section{Age Distribution}

Majority of patients were in the age group of 61$70(46.7 \%)$ year, the mean age of patients with multiple myeloma in our study was 59.2 with a standard deviation of 8.19 years, and median age of 60 years. By observing these results, it seems that multiple myeloma has younger age predominance in Indian population compared to western population.

\section{Gender Distribution}

In our study we found that incidence is more among males (70\%) as compared to females $(30 \%)$. Sex ratio in our study is found to be 2.3:1.
This correlates with the fact that incidence rates are higher among men than women

\section{Clinical Features.}

The most common presenting complaint in our study was back pain $(73.3 \%)$ followed by pathological fractures and paraparesis. In our study vast majority of patients in addition, had nonspecific complaints like fatigue (63.3\%) weight loss $(83.3 \%)$ generalized weakness $(86.7 \%)$

\section{Lab Parameters}

The findings in current study resembles results of studies conducted on Indian sub-population, whereas are slightly different from western studies. From the above results we can see that hypercalcemia is more common and anemia is less common in Indian population as compared to western population.

\section{Serum/Urine Electrophoresis}

In our study $93.3 \%$ patients had M-protein in their blood; Kappa subtype was more common (56.7\%) than lambda (43.3\%).

\section{Staging}

Most of the patients in our study (43.3\%) were in stage II at presentation according to international staging system. However adequate data was not available (because some patients did not have beta 2 macroglobulin levels report) to compare with other studies. $94 \%$ of the patients had M-protein in their blood.

\section{Response to Chemotherapy}

Five different combinations of chemotherapeutic agents were used in current study they are Bortezomib+ Dexamethasone (60\%), Bortezomib+ Dexamethasone+ Lenalidomide (16\%), Bortezomib+ Cyclophosphamide + Dexamethasone (10\%), Bortezomib+ Dexamethasone+ Pomalidomide (7\%), Bortezomib+ Dexamethasone+ Thalidomide (7\%).

\section{a. Remission}

Newer chemotherapeutic agents have given better response rates when compared to older regimens. In our study $30 \%$ of patients achieved remission in 10-12 months and $27.7 \%$ in12-18 months. 
However not all patients in the study achieved remission because some patients who enrolled later in the study were still on induction regimens. Time for remission ranged from 9 months to 30 months, and mean time for remission was 16.12 months in our study. Older regimens as compared to newer regimens, takes longer period for attaining remissions and have shorter duration of overall survival. When we look at individual regimens, in our study Bortezomib+ Cyclophosphamide + Dexamethasone had lowest mean time for remission followed by Bortezomib + Dexamethasone.

\section{b. Complications of chemotherapy.}

In current study, most common complication was peripheral neuropathy accounting for $20 \%$ followed byinfections, relapse and thrombosis.

1. I.INFECTION was more common in Bortezomib +Cyclophosphamide+Dexamethasone regimen. Both the patients on this regimen had Urinary tract infections. One patient on Bortezomib + Dexamethasone regimen developed pneumonia. This is probably because of higher dose of steroid used and increased predisposition of cystitis by cyclophosphamide.

2. THROMBOSIS was common in patients on Dexamethasoneplus thalidomide/ lenalidomide regimen in current study. 2 out of 30 patients developed deep vein thrombosis of lower limb.

3. PERIPHERAL NEUROPATHY In our study peripheral neuropathy was more commonly seen in patients on Bortezomib + Dexamethasone regimen as compared to bortezomib with thalidomide. This is in contrast to Cömerte et $\mathrm{al}^{2}$ who found higher incidence of peripheral neuropathy in thalidomide-based regimens. This could be because of confounding factor of underlying comorbidities (diabetes in our patient) which contributed to peripheral neuropathy.

4. RELAPSE In our study two patients treated with Bortezomib +Dexamethasone regimen had relapsed after achievingremission and one patient relapse on Bortezomib +Cyclophosphamide +Dexamethasone regimen. Evenother studies 1,3 have reported higher chances of relapse with Bortezomib + Cyclophosphamide + Dexamethasone regimens compared to lenalidomide or pomalidomide with bortezomib. When compared with older chemotherapeutic regimens, relapse rates and infection rates are significantly lower, whereas thrombosis and peripheral neuropathy rates are higher with newer regimens

\section{Graph showing distribution of Age Group}

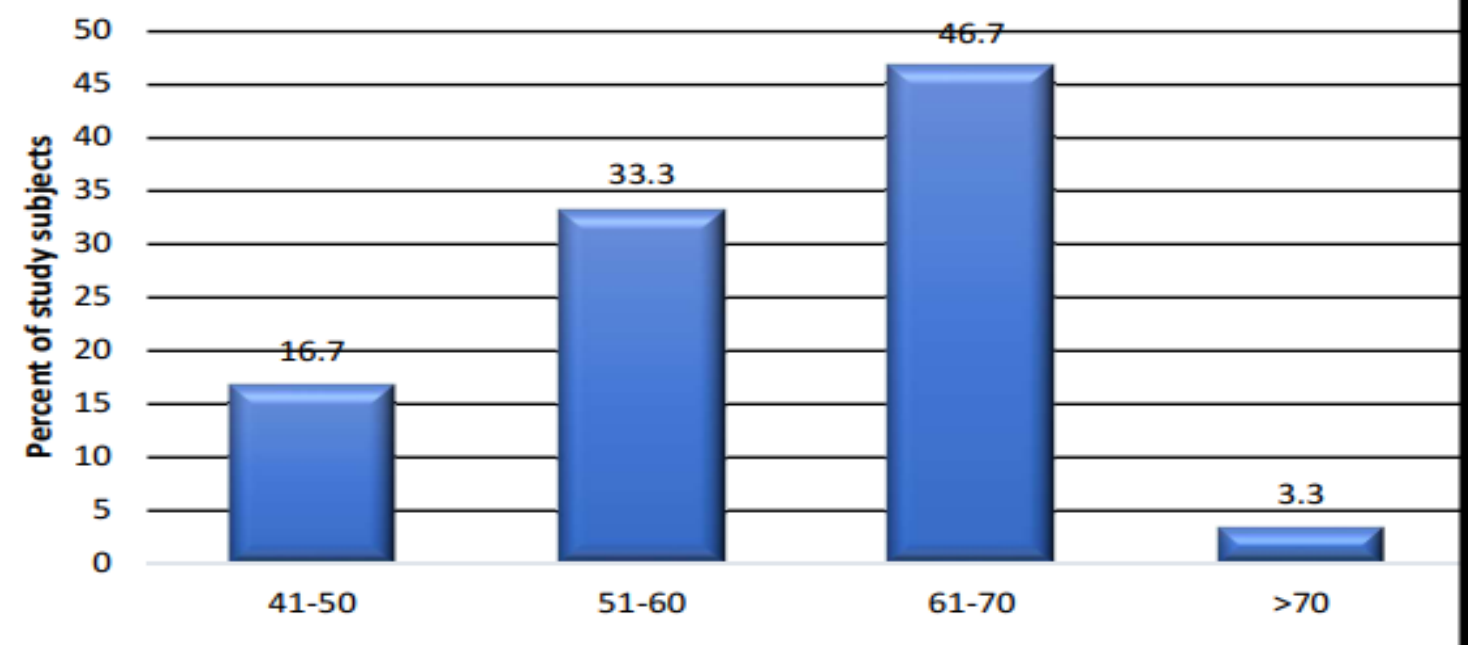

Age of the study subjects (in years) 


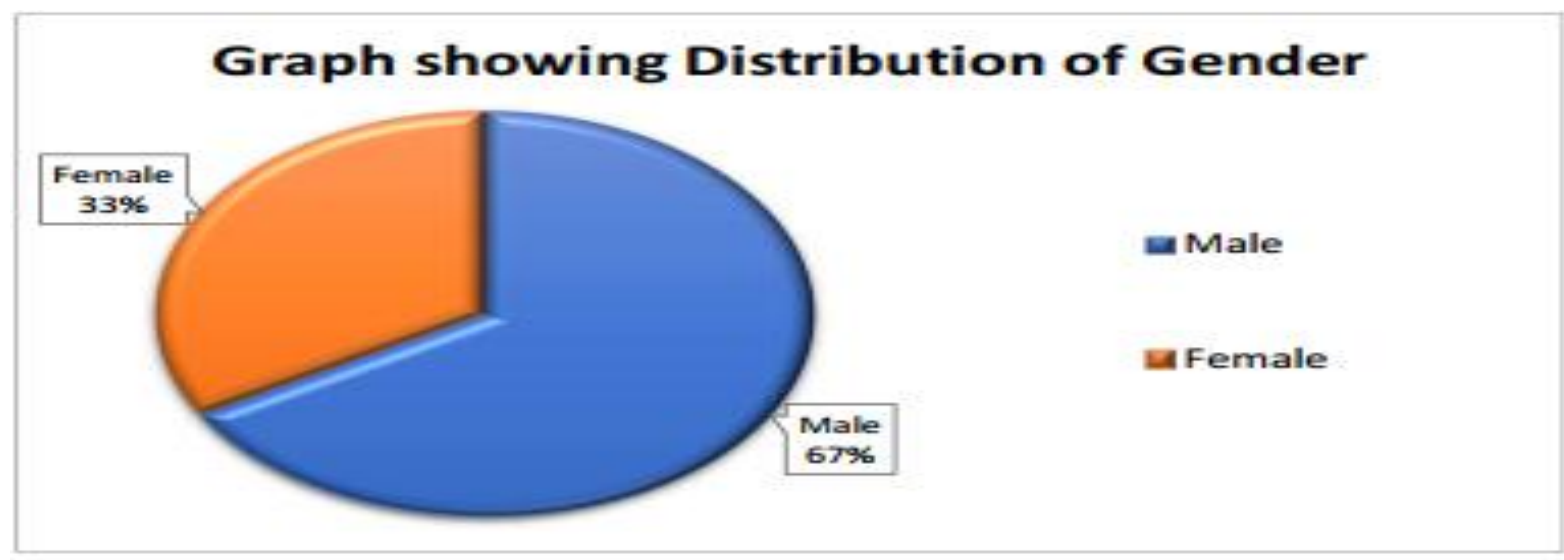

$70 \%$ of affected patients were ales and $30 \%$ were females.

Males were affected more than females. Male female ratio is $2.3: 1$.

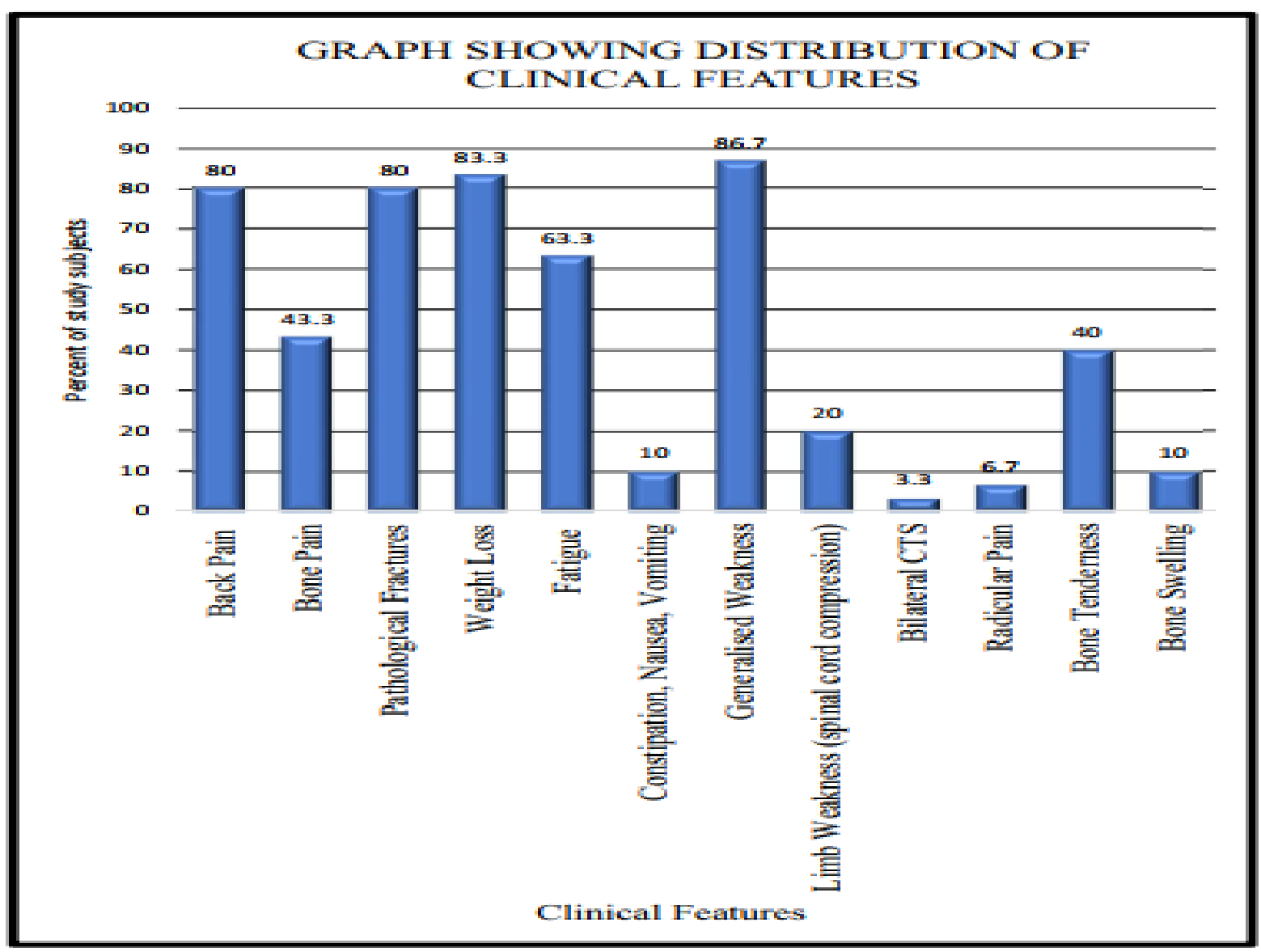



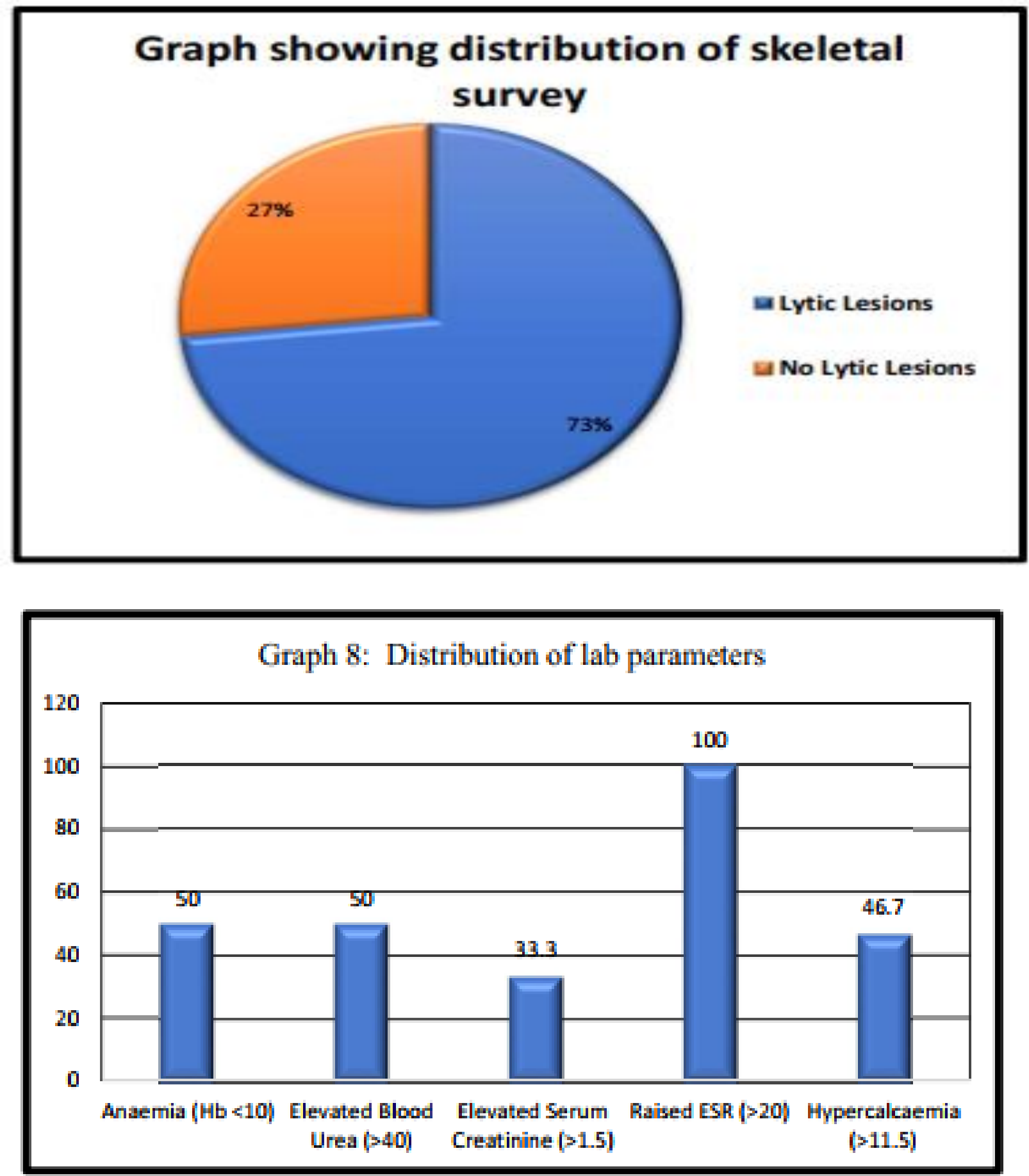

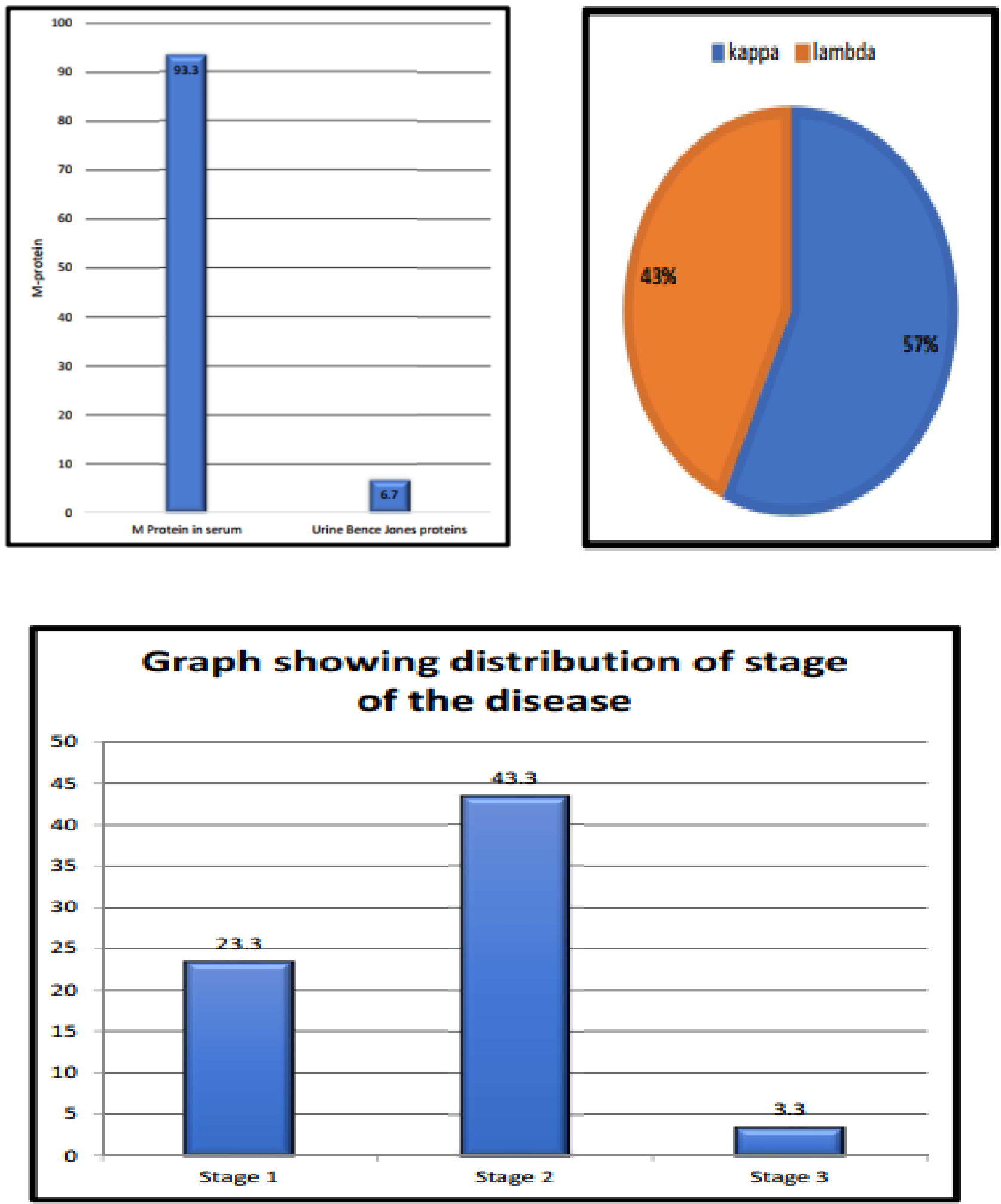

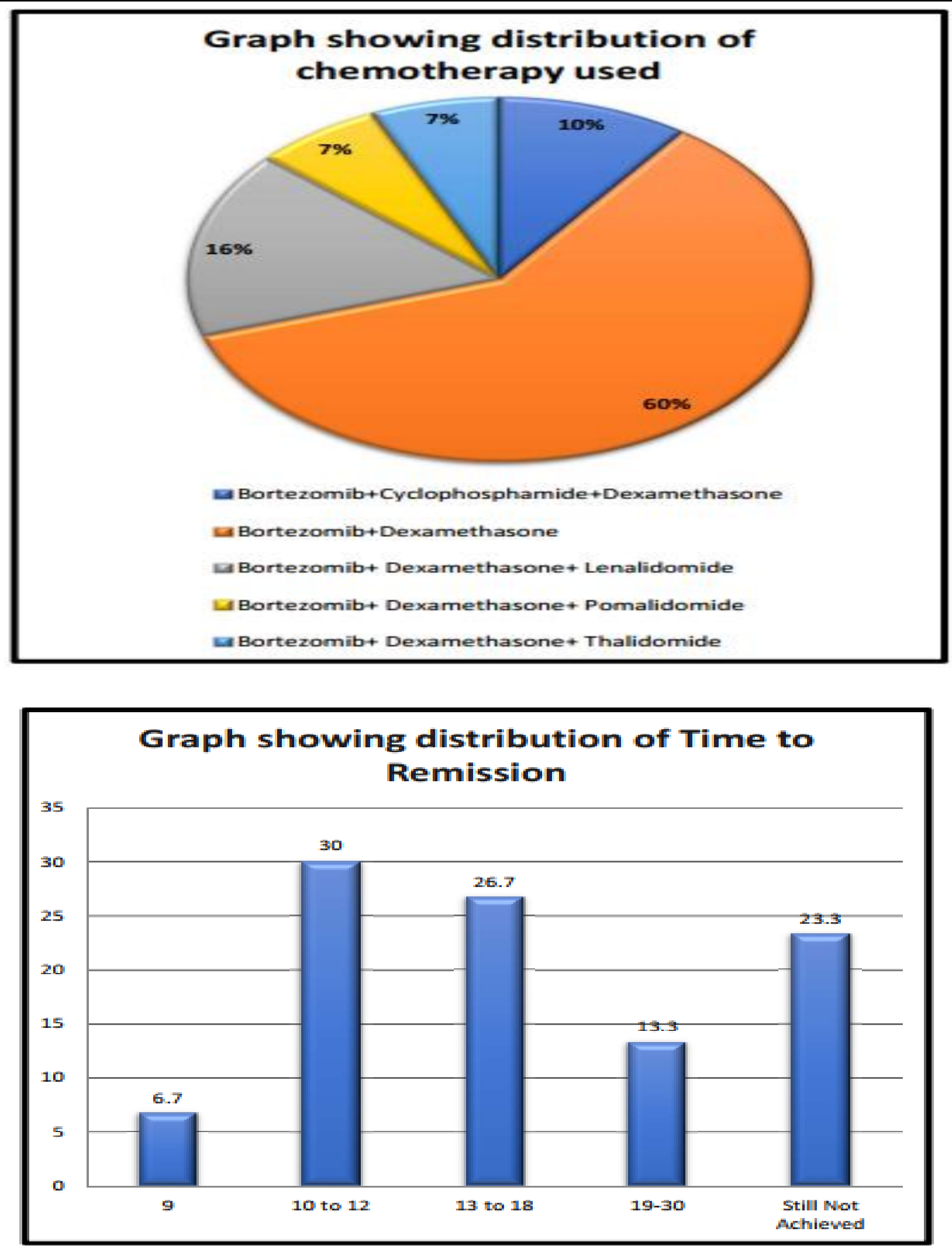

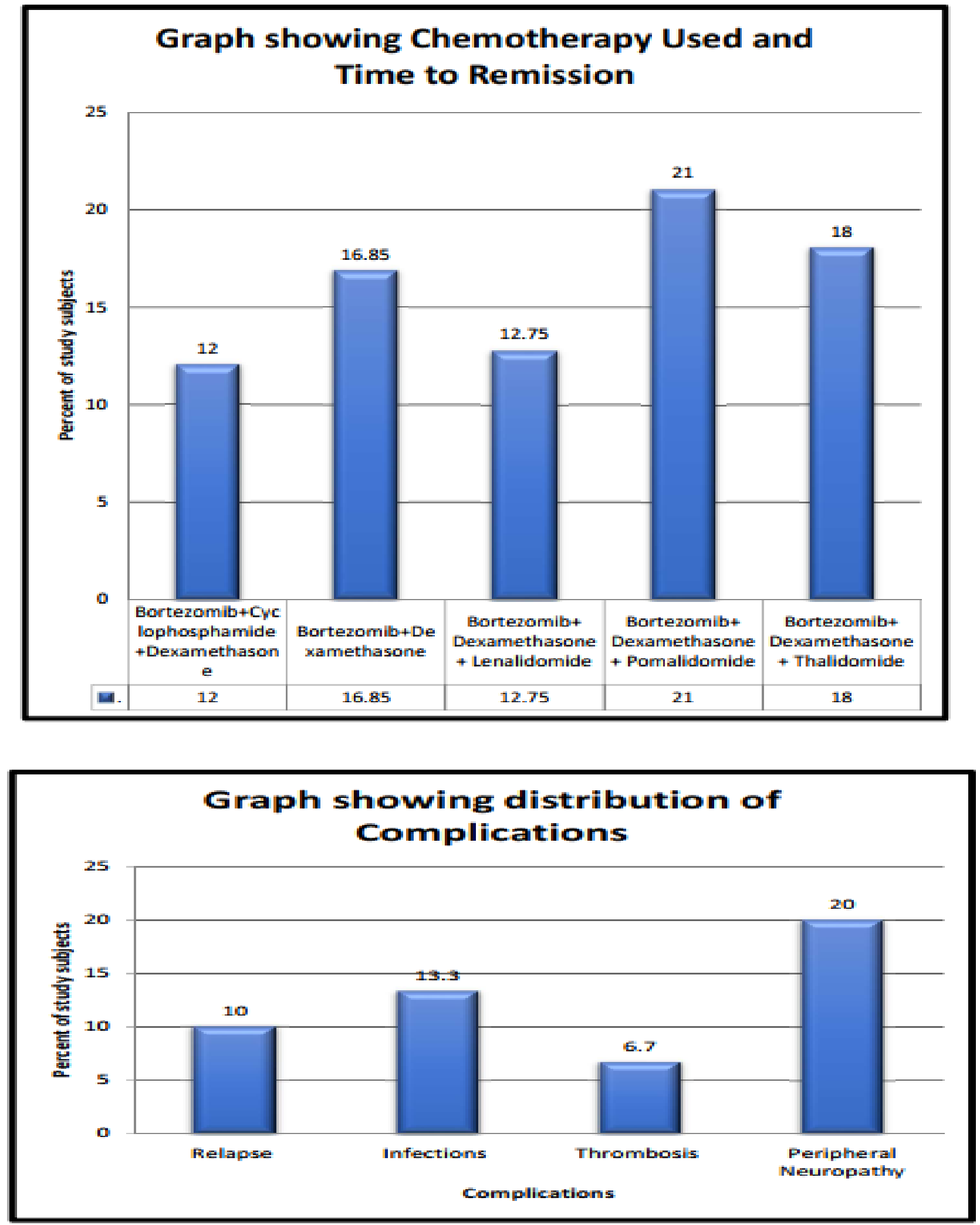


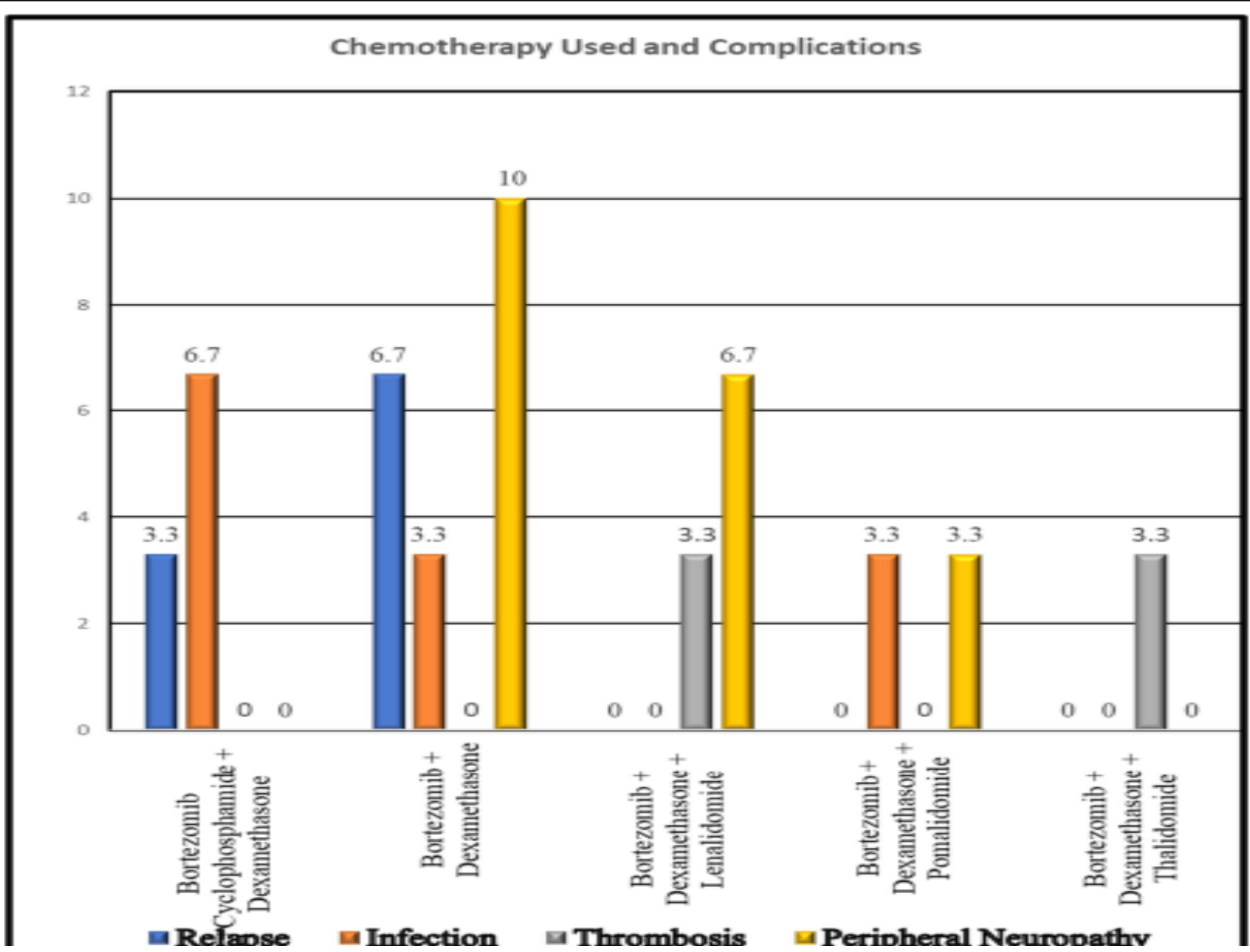

Table showing comparison between different chemotherapeuticregimens in terms of relapse infection thrombosis and peripheralneuropathy. 1

\section{Discussion}

From the current study we can see that multiple myeloma is commonly presets in $7^{\text {th }}$ decade, with mean age of 59 years and a decade earlier in Indian population as compared to the world mean age of presentation and has male preponderance.

Most common presenting symptom is back pain, although many patients had nonspecific complaints. The incidence of hypercalcemia is comparatively higher but anemia and renal failure is lesser in our patients than western countries. Better response is observed with the newer chemotherapy agents in terms of time required for remission and lesser incidence of relapse and drug related complications like infections

\section{Conclusion}

Multiple myeloma is a potentially curable disease. Early detection and treatment with newer advanced therapies gives better quality of life.

\section{Limitations}

Smaller number of study subjects. Patients enrolled during later part of study were stillon induction regimens hence couldn't assess for remission. Complications of specific chemotherapeutic agents like peripheral neuropathy could be due to underlying comorbidities. Relapse in few study subjects was due to noncompliance with treatment. We could not follow-up all the patients for longer period to see long term effect of different chemotherapeutic regimens. Further follow-up till the terminal events in these patients is required to fully evaluate the impact of newer therapies on quality of life and lifespan itself

\section{References}

1. Hematology, C. Wintrobe's Clinical Hematology. 
2. Cömert, M., Güneş, A. E., Şahin, F. \& Saydam, G. Multiple myeloma dayaamkalitesivedestektedavisi. Turkish J. Hematol. 30, 234-246 (2013).

3. Dex, R. E. V et al. Combination therapy with lenalidomide plus dexamethasone (rev/dex) for newly diagnosed myeloma. (2005). doi:10.1182/blood-2005-07-2817

4. Ttal, M. I. A., Arousseau, J. E. A. N. U. C. H., Toppa, A. N. N. E. A. S. \& Otto, J. E. A. N. A. S. A Prospective, Randomized Trial Of Autologous Bone Marrow Transplantation And Chemotherapy In Multiple Myeloma. 91-97 (1996).

5. Myeloma, N. D. Newly Diagnosed Myeloma.

6. Palumbo, A. NIH Public Access. 112, 1522-1528 (2009).

7. Dimopoulos MA, Anagnostopoulos A, Weber D. Treatment of plasma cell dyscrasias with thalidomide and its derivatives. J Clin Oncol. 2003;21:44444454.

8. Field-smith, A. Bortezomib (VelcadeTM) in the treatment of multiple myeloma. 2, 271-279 (2006)

9. Richardson PG, Blood E, Mitsiades CS, et al. A randomized phase 2 study of lenalidomide therapy for patients with relapsed or relapsed and refractory multiple myeloma Blood. 2006;108:34583464.

10. Rajkumar SV, Hayman SR, Lacy MQ, et al. Combination therapy with lenalidomide plus dexamethasone (Rev/Dex) for newly diagnosed myeloma. Blood. 2005;106: 4050-4053.

11. Dimopoulos M, Spencer A, Attal M, et al. Lenalidomide plus dexamethasone for relapsed or refractory multiple myeloma . N Engl J Med. 2007;357:2123-2132.

12. Weber DM, Chen C, Niesvizky R, et al. Lenalidomide plus dexamethasone for relapsed multiple myeloma in North
America. N Engl J Med. 2007;357:21332142.

13. Boccadoro M, Palumbo A. New developments and treatment in multiple myeloma: New drugs in the treatment of multiple myeloma. Ann Oncol. 2002;13(SUPPL. 4):55-8.

14. Major P, Lortholary A, Hon $\mathrm{J}$ et al. Zoledronic acid is superior to pamidronate in the treatment of hypercalcemia of malignancy: a pooled analysis of two randomized, controlled clinical trials. $\mathrm{J}$ Clin Oncol 2001; 19: 558-567.

15. Berenson JR, Lichtenstein A, Porter L et al. Efficacy of pamidronate in reducing skeletal events in patients with advanced multiple myeloma. Myeloma Aredia Study Group. N Engl J Med 1996; 334: 488-493.

16. Rosen LS. Zoledronic acid versus pamidronate in the treatment of skeletal metastases in patients with breast cancer or osteolytic lesions of multiple myeloma : a phase III, doubleblind, comparative trial. Cancer J 2001; 7: 377-387.

17. Khalil Al-Farsi et al, Oman Medical Journal (2013) Vol. 28, No. 1:3-11

18. Kyle RA, Therneau TM, Rajkumar SV, Larson DR, Plevak MF, Melton LJ III. Incidence of multiple myeloma in Olmsted County, Minnesota: trend over 6 decades. Cancer. 2004;101:2667-2674.

19. Lewis DR, Pottern LM, Brown LM, et al. Multiple myeloma among blacks and whites in the United States: the role of chronicantigenic stimulation. Cancer Causes Control. 1994;5:529-539.

20. Abergel, R. \& Gross, E. www.aging us.com AGING 2017 Vol. 9, No. 11. 9, 2241-2242 (2017).

21. Athira et al. Clinical Profile Of Multiple Myeloma At GRH - Madurai 2005 - 2007

22. Article, O. Patterns of Multiple Myeloma During the Past 5 Decades: Stable Incidence Rates for All Age Groups in the Population but Rapidly Changing Age 
Distribution in the Clinic. 225-230 (2010). doi: $10.4065 / \mathrm{mcp} .2009 .0426$

23. Gerecke, C. et al. The Diagnosis and Treatment of Multiple Myeloma. 470-477 (2016). doi:10.3238/arztebl.2016.0470S

24. Naryan, R. \& Ali, M. Clinical Profile of Multiple Myeloma in South India. 62-66 (2018). doi:10.4103/ijmpo.ijmpo. 